\title{
A planar metamaterial: Polarization independent fishnet structure
}

\author{
Kamil Boratay Alici ${ }^{a, b, *}$, Ekmel Ozbay ${ }^{a, b, c}$ \\ ${ }^{a}$ Nanotechnology Research Center, Bilkent University, 06800 Cankaya, Ankara, Turkey \\ ${ }^{\mathrm{b}}$ Department of Physics, Bilkent University, 06800 Ankara, Turkey \\ ${ }^{\mathrm{c}}$ Department of Electrical and Electronics Engineering, Bilkent University, 06800 Ankara, Turkey
}

Received 18 July 2007; received in revised form 2 January 2008; accepted 10 January 2008

Available online 18 January 2008

\begin{abstract}
We numerically and experimentally investigate a planar metamaterial that is composed of connected cut-wire pairs and continuous wires operating at $21 \mathrm{GHz}$. The characterization was performed by using the effective medium theory. The existence of negative refraction is concluded from the transmission data of four structures: cut-wire pairs, shorted cut-wire pairs, composite metamaterial, and shorted composite metamaterial.
\end{abstract}

(C) 2008 Elsevier B.V. All rights reserved.

PACS : $41.20 . \mathrm{Jb}$

Keywords: Planar; Metamaterial; Fishnet; Polarization independent; Microwave

\section{Introduction}

An artificial medium composed of periodically arranged electrically small resonators can exhibit an effective negative index of refraction. Experimental demonstration of the medium called an electromagnetic metamaterial was conducted by using split ring resonators (SRRs) and continuous wires [1,2]. The periodic arrangement of thin wires provides a negative effective permittivity at frequencies lower than the plasma frequency of the system [3]. The SRRs provide a strong magnetic resonance that leads to a negative effective permeability medium $[4,5]$.

\footnotetext{
* Corresponding author at: Nanotechnology Research Center, Bilkent University, 06800 Cankaya, Ankara, Turkey.

Tel.: +90 312290 1018; fax: +90 3122901015 .

E-mail address: bora@fen.bilkent.edu.tr (K.B. Alici).
}

One of the major aims of the study of metamaterials is to achieve negative refraction at the visible range of the electromagnetic spectrum. By scaling the size of the SRR, a magnetic response at around $1 \mathrm{THz},[6] 6 \mathrm{THz}$, [7] $70 \mathrm{THz}$, [8] $100 \mathrm{THz}$, [9] and $200 \mathrm{THz}$ [10] is achieved experimentally. However, metamaterials that are composed of layers of SRRs and wires have several restrictions. Firstly, as the size of the SRR is decreased, the magnetic resonance becomes weaker and approaches a limit [11]. A large number of SRR layers have to be produced and stacked in order to obtain the negative index medium. The fabrication of SRRs with resonance frequency at the visible range is rather difficult. Moreover, the alignment of the produced layers is a major problem. The restrictions of the conventional metamaterial construction are overcome by way of the introduction of planar metamaterials [10-14].

Metamaterials for which the propagation direction is normal to the plane of the metamaterial layers are called 
planar metamaterials. A negative index metamaterial is experimentally demonstrated at $150 \mathrm{THz}$ by using a pair of metal layers that are separated by a dielectric in order to provide resonant interactions along with a periodic array of holes [12] and at $200 \mathrm{THz}$ by using a thin layer of pairs of parallel metal nanorods [13]. At this point, it is noteworthy that the imaginary part of the effective index of refraction for these works at the operation frequency was large, and thereby, the losses were significant. The systematic study and characterization of cut-wire pairs (cwp) operating at $300 \mathrm{THz}$ [14] and the fishnet design at $200 \mathrm{THz}$ [15] were performed experimentally. In the fishnet design, the cut-wire pairs are as wide as the lattice constant and are physically connected with the continuous wires, see Fig. 1. Recently, a negative index metamaterial operating at $385 \mathrm{THz}$ was experimentally demonstrated by using the fishnet design [16].

The experimental characterization and parametric study of planar metamaterials is rather easy for the operation frequencies at the $\mathrm{GHz}$ range. One advantage of working at this frequency regime is that the metamaterial features are sufficiently large to study several effects, such as the misalignment of metal pairs and metamaterial layers. Such studies are rather difficult to perform at the $\mathrm{THz}$ range. Recently, planar metamaterials at $14 \mathrm{GHz}$ attained by using cut-wire pairs [17,18], and at $13 \mathrm{GHz}$ by using the fishnet design [19], were demonstrated experimentally. In the present

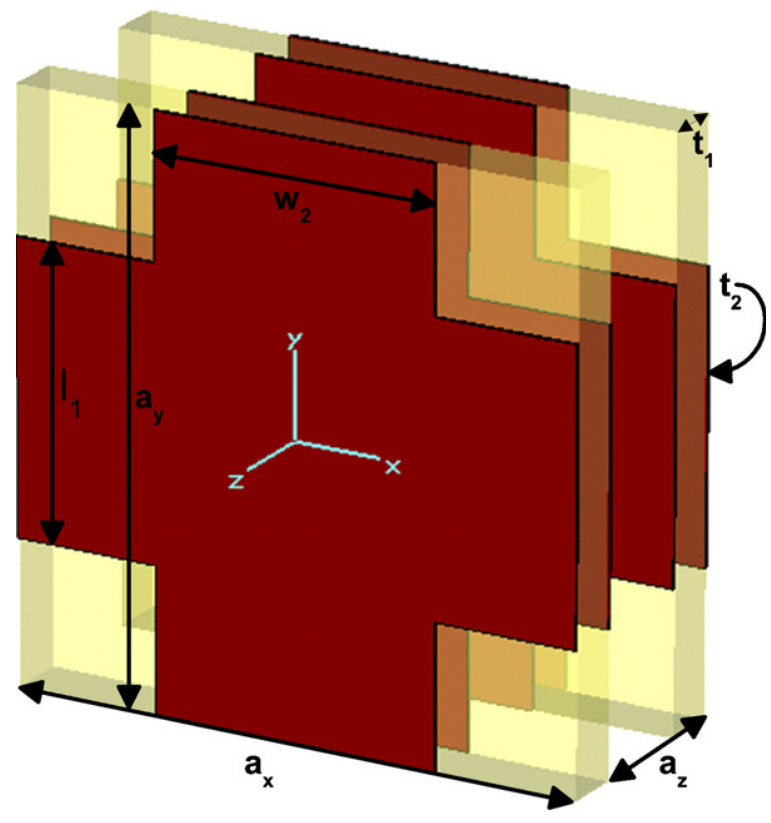

Fig. 1. The geometry of one unit cell of the fishnet metamaterial. The electromagnetic wave propagates in the $-z$ direction, in which $E$ and $B$ are along the $y$ and $z$ directions. There are two layers in the propagation direction; the parameters are given in the text. work, we extend the experimental and numerical study of the fishnet structure to the case in which the structure is symmetric with respect to the $x= \pm y$ plane, which is shown in Fig. 1. In this case, the $x$ - and $y$-polarized incident wave the structure gives the same response.

\section{Theory and simulations}

Theoretical modeling of the metamaterials is performed by using effective inductor-capacitor circuits (LC circuit). When the elements of the metamaterial in the propagation direction are small compared to the free space wavelength at the operation frequency, we can use the quasistatic process approach [20]. An LC circuit model for the fishnet structure was given in Ref. [17]. The model predicts the dependence of magnetic resonance frequency on the structure's parameters by way of the formula:

$$
f_{\mathrm{m}}=\sqrt{\frac{1}{l_{1}^{2}}+\frac{w_{2}}{l_{1} l_{2} a_{x}},}
$$

where $l_{2}=\left(a_{y}-l_{1} / 2\right), l_{1}$ is the cut-wire length, $w_{2}$ the wire width, and $a_{x}$ and $a_{y}$ are the periodicity in the $x$ and $y$ directions, see Fig. 1 .

There are two methods for the characterization of metamaterial slabs: the retrieval procedure and the effective medium analysis. The retrieval of the effective index of refraction $(\mathrm{n})$, impedance $(\mathrm{z})$, permittivity $(\varepsilon)$, and permeability $(\mu)$ of a metamaterial slab is extracted from the magnitude and phase of the reflection and transmission data [21,22]. On the other hand, the effective medium analysis is performed by only using the magnitude of the transmission data. However, four different structures are necessary: $\mu$-negative (MNG) material, shorted MNG material, composite medium of MNG materials, and continuous wires ( $\mathrm{cmm}$ ), as well as the shorted cmm. [23] In the present work, we followed the latter characterization method, in which our MNG material is the cut-wire pairs, Fig. 2a, and the $\mathrm{cmm}$ is the fishnet structure, Fig. 3a.

We perform a qualitative effective medium theory characterization numerically via the commercial software CST Microwave Studio. This software is a three dimensional full-wave solver that employs the finite integration technique [24]. In the simulations, we insert the unit cell of the structure in a waveguide and obtain the scattering parameters by using waveguide ports. By way of this method, the structure unit cell is assumed to extend to infinity in the lateral directions and the incident, reflected and transmitted waves are in the form of a plane wave. The $\mathrm{E}$ field is in the $y$-direction, the $\mathrm{B}$ 
(a)

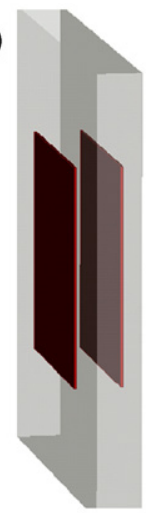

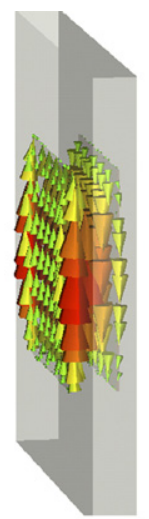

$\mathrm{A} / \mathrm{m}$

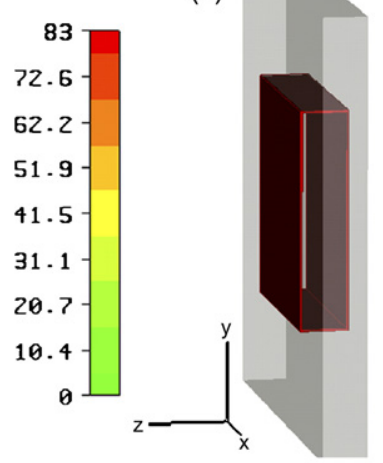

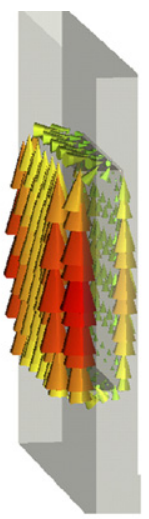

A/m

21.1

18.4

15.8

13.2

10.5

7.9

5.27

2.63

0

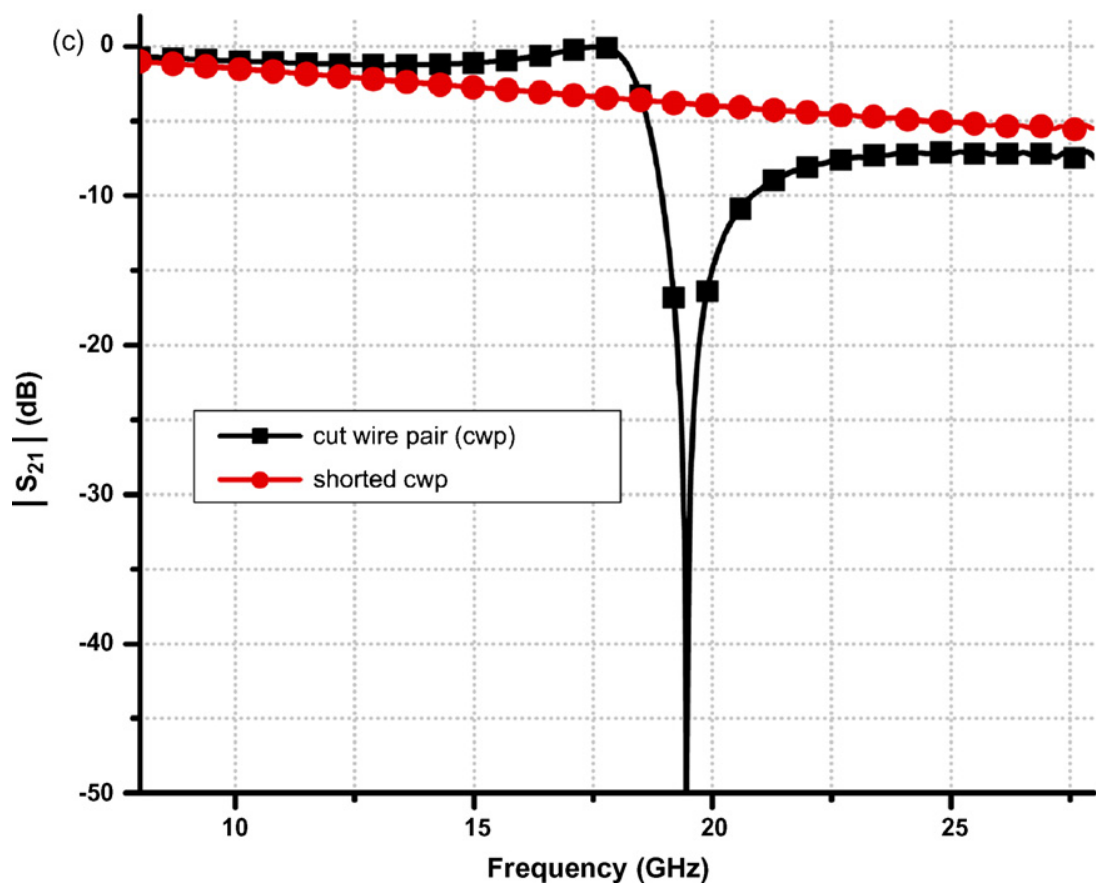

Fig. 2. The geometry and surface current: (a) the cut-wire pair (cwp); (b) shorted cut-wire pair; (c) transmission spectrum magnitude of the cwp and shorted cwp structures.

field is in the $x$-direction, and the propagation vector is in the negative $z$-direction: $\hat{E}=\hat{y}, \hat{H}=\hat{x}, \hat{k}=-\hat{z}$. The metallic features are assumed to be a perfect electric conductor (PEC) and the relative permittivity of the substrate is $\varepsilon_{\mathrm{r}}=1.94$. Other structure parameters are as follows, see Fig. 1: $a_{x}=a_{y}=10 \mathrm{~mm}, a_{z}=3 \mathrm{~mm}$, $l_{1}=w_{2}=5 \mathrm{~mm}, t_{1}=1 \mathrm{~mm}, t_{2}=0.05 \mathrm{~mm}$. We have one layer of the metamaterial in the propagation direction, in which the metal and substrate losses are ignored. The results of the first part of the effective medium analysis are summarized in Fig. 2.

For the case of metamaterials a stop band at the transmission spectra indicates a resonance phenom- enon, which could be electrically or magnetically originated. The fishnet metamaterial is composed of metallic and dielectric parts both of which are nonmagnetic. The only source of magnetic resonance is the circulating currents driven by the capacitance between the cut wires. A simple method to determine whether a stop band is due to the magnetic or electric resonance is to shorten the cut wire capacitance as shown in Fig. 2b. By this way the driven force of the circulating currents will be eliminated and the magnetically originated stop band at the transmission spectra will disappear, Fig. 2c [23]. Moreover, as we kill the magnetic resonance by shorting the cut-wire pairs 

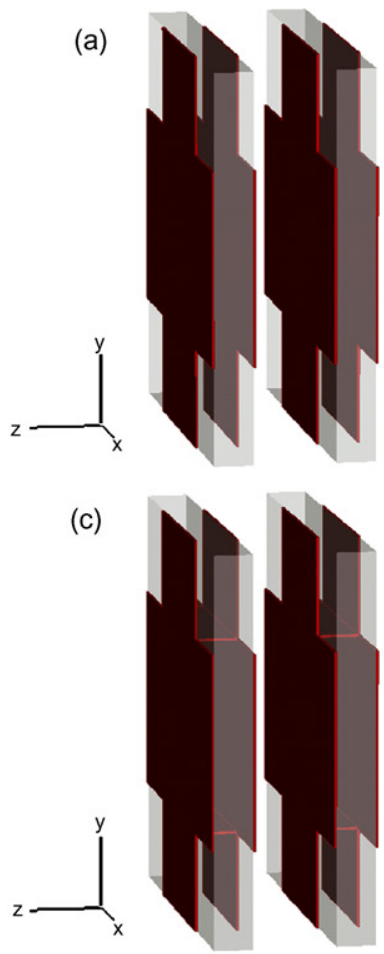

(b)

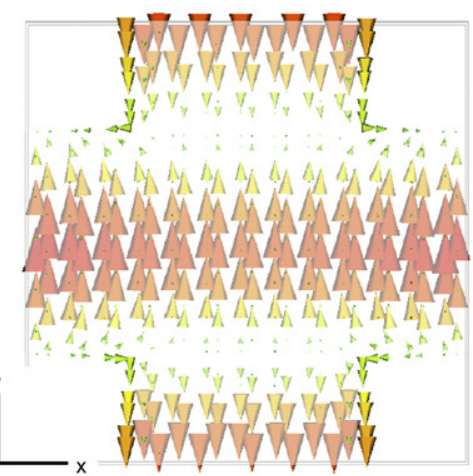

(d)

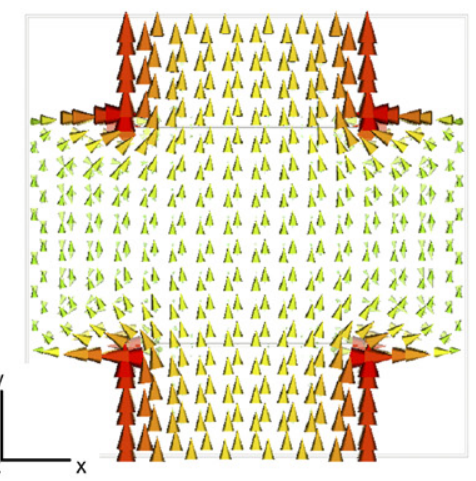

$\mathrm{A} / \mathrm{m}$ $\left.\begin{array}{r}94.4 \\ 82.6 \\ 70.8 \\ 59 \\ 47.2 \\ 35.4 \\ 23.6 \\ 11.8 \\ 0\end{array}\right]$

$\mathrm{A} / \mathrm{m}$

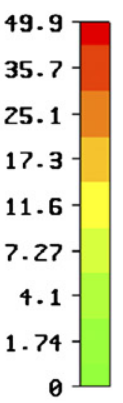

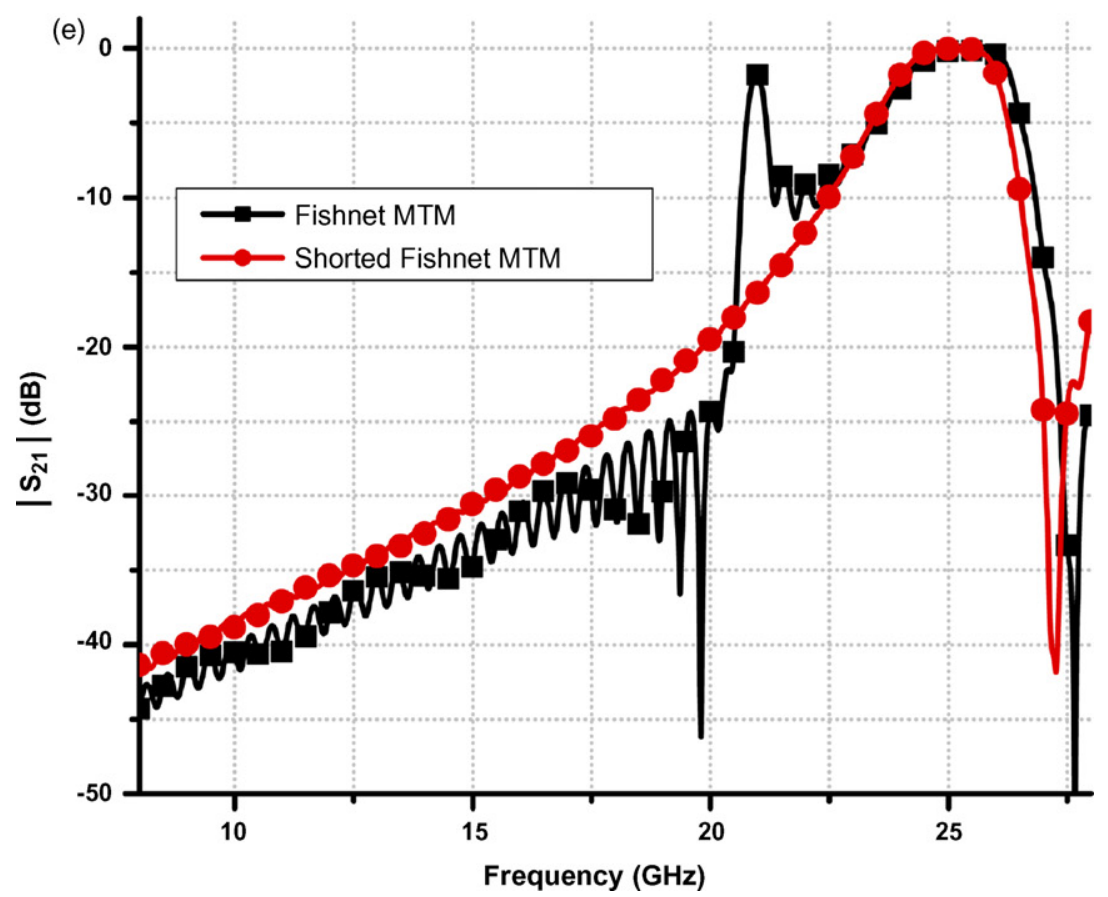

Fig. 3. Schematic view: (a) two layer $\mathrm{cmm}$; (c) two layer shorted $\mathrm{cmm}$. Surface current on the face of the first layer: (b) $\mathrm{cmm}$; (d) shorted $\mathrm{cmm}$. (e) Magnitude of the transmission data for the $\mathrm{cmm}$ and shorted $\mathrm{cmm}$ structures.

the circulating surface current in Fig. 2a disappears and the current pattern becomes similar to an electric dipole, Fig. 2b. The second part of the effective medium theory analysis is summarized in Fig. 3. The geometry of the $\mathrm{cmm}$ and shorted $\mathrm{cmm}$ are shown in Fig. $3 \mathrm{a}$ and $\mathrm{c}$, respectively. In this part, there are two layers in the propagation direction. The transmission data implies the existence of a negative index of refraction at around 

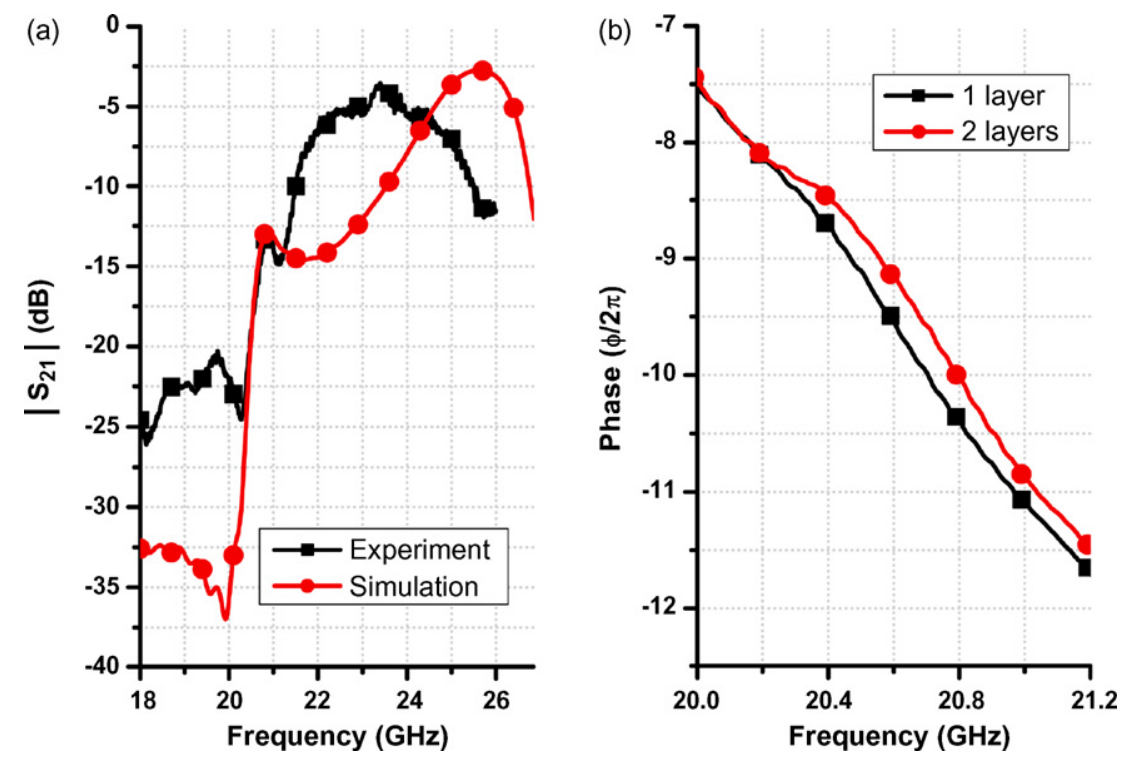

Fig. 4. (a) The transmission spectrum of the fishnet metamaterial simulation and experiment. In the simulation, the loss of the metal and dielectric parts is taken into account. (b) Phase spectra of the metamaterial for a different number of layers.

$21 \mathrm{GHz}$. However, the magnetic resonance frequency of the cut-wire pairs was around $19.5 \mathrm{GHz}$. This difference is a characteristic of the fishnet metamaterial and can be explained by considering the surface current at the first face of the metamaterial, Fig. 3b. When we combine the cut-wire pairs with the continuous wires, the effective cut-wire length decreases and the magnetic resonance frequency increases. Finally, we would like to note that the transmission properties remain the same when the incident wave polarization is rotated $90^{\circ}$. Since the structure is symmetric with respect to the $x= \pm y$ plane this behavior was expected.

\section{Experiment}

The experiments were performed via an HP8510C Network Analyzer and two standard gain horn antennae. After the full two-port calibration we first measured the scattering parameters for the free space, i.e. without the metamaterial layers being inserted. Subsequently, we repeat the experiment wherein the metamaterial slab is in between the antennae. The distance between the transmitter and receiver antennae is kept fixed at $79 \mathrm{~mm}$ during the experiments. The metamaterial substrate is cardboard of $1 \mathrm{~mm}$ thickness and the metallic features are formed by using an aluminum based tape. In Fig. 4, the transmission magnitude and phase data are shown. The transmission data is scaled to the free space data. The experimental results are in good agreement with the simulation. At this point we should clarify the difference between the design simulations shown in
Fig. 3e and the more realistic simulation shown in Fig. 4a. In the design simulations, we assumed lossless metal and dielectric parts in order to clearly demonstrate the effective medium theory concepts (see Figs. $2 \mathrm{c}$ and $3 \mathrm{e}$ ). In Fig. 4a, when comparing the simulation and experimental results we have taken the loss effects into account. The conductivity of the aluminum type and dielectric were 20,000 and $0.001 \mathrm{~S} / \mathrm{m}$, respectively. The possible reasons of the discrepancies between the simulation and experiment are: the misalignment of the continuous and cut-wire pairs in a layer, small deviations of the fabricated material parameters from the intended values and misalignment of the multiple layers. We expect the transmission band between the frequencies 20.2 and 21.2 is negative (see Figs. $3 e$ and 4a). The fractional bandwidth of the negative region is narrow and calculated as $2 \%$ by using the formula: $\mathrm{FBW}=\Delta f / f_{0}$, where is $\Delta f$ is the half power bandwidth and $f_{0}$ is the center frequency. The effective medium theory is a qualitative approach and in order to determine the negative band exactly a robust retrieval analysis is necessary [21,22]. In Fig. 4b, we demonstrate that the phase shift of the wave within the left-handed band is negative. In this band, as the number of layers increase, the phase decreases.

\section{Conclusion}

In summary, we characterized a planar metamaterial operating at $21 \mathrm{GHz}$ by using a qualitative effective medium theory. The planar metamaterial was the fishnet 
structure, which is symmetric with respect to the $x= \pm y$ plane. The operation frequency of the fishnet metamaterial is higher than the corresponding cut-wire pair magnetic resonance frequency. The left-handed nature of the transmission peak is identified unambiguously by using the shorted $\mathrm{cmm}$ structure. The experimental phase data strengthens the indication of the negative index of refraction. By investigating the planar metamaterials at microwave frequencies several contributions can be added to the study of metamaterials at optical frequencies.

\section{Acknowledgements}

This work is supported by the European Union under the projects EU-NoE-METAMORPHOSE, EU-NoEPHOREMOST, and TUBITAK under the Project nos. 105E066, 105A005, 106E198 and 106A017. One of the authors (E.O.) also acknowledges partial support from the Turkish Academy of Sciences.

\section{References}

[1] D.R. Smith, W.J. Padilla, D.C. Vier, S.C. Nemat-Nasser, S. Schultz, Phys. Rev. Lett. 84 (2000) 4184.

[2] R.S. Penciu, M. Kafesaki, T.F. Gundogdu, E.N. Economou, C.M. Soukoulis, Photon. Nanostruct. Fund. Appl. 4 (2006) 12.

[3] J.B. Pendry, A.J. Holden, W.J. Stewart, I. Youngs, Phys. Rev. Lett. 76 (1996) 4773.

[4] J.B. Pendry, A.J. Holden, D.J. Robbins, W.J. Stewart, IEEE Trans. Microwave Theory Tech. 47 (1999) 2075.

[5] L. Zhang, G. Tuttle, C.M. Soukoulis, Photon. Nanostruct. Fund. Appl. 2 (2006) 155.

[6] T.J. Yen, W.J. Padilla, N. Fang, D.C. Vier, D.R. Smith, J.B. Pendry, D.N. Basov, X. Zhang, Science 303 (2004) 1494.
[7] N. Katsarakis, G. Konstantinidis, A. Kostopoulos, R.S. Penciu, T.F. Gundogdu, M. Kafesaki, E.N. Economou, T. Koschny, C.M. Soukoulis, Opt. Lett. 30 (2005) 1348.

[8] S. Zhang, W. Fan, B.K. Minhas, A. Frauenglass, K.J. Malloy, S.R.J. Brueck, Phys. Rev. Lett. 94 (2005) 037402.

[9] S. Linden, C. Enkrich, M. Wegener, J. Zhou, T. Koschny, C.M. Soukoulis, Science 306 (2004) 1351

[10] C. Enkrich, M. Wegener, S. Linden, S. Burger, L. Zschiedrich, F. Schmidt, J.F. Zhou, T. Koschny, C.M. Soukoulis, Phys. Rev. Lett. 95 (2005) 203901.

[11] M.W. Klein, C. Enkrich, M. Wegener, C.M. Soukoulis, S. Linden, Opt. Lett. 31 (2006) 1259.

[12] S. Zhang, W. Fan, N.C. Panoiu, K.J. Malloy, R.M. Osgood, S.R.J. Brueck, Phys. Rev. Lett. 95 (2005) 137404.

[13] V.M. Shalaev, W. Cai, U.K. Chettiar, H. Yuan, A.K. Sarychev, V.P. Drachev, A.V. Kildishev, Opt. Lett. 30 (2005) 3356.

[14] G. Dolling, G. Enkrich, M. Wegener, J.F. Zhou, C.M. Soukoulis, S. Linden, Opt. Lett. 30 (2005) 3198.

[15] S. Zhang, W. Fan, K.J. Malloy, S.R.J. Brueck, N.C. Panoiu, R.M. Osgood, Opt. Express 13 (2005) 4922.

[16] G. Dolling, M. Wegener, C.M. Soukoulis, S. Linden, Opt. Lett. 32 (2007) 53.

[17] K. Guven, M.D. Caliskan, E. Ozbay, Opt. Express 14 (2006) 8685.

[18] J. Zhou, L. Zhang, G. Tuttle, T. Koschny, C.M. Soukoulis, Phys. Rev. B 73 (2006) 041101

[19] M. Kafesaki, I. Tsiapa, N. Katsarakis, Th. Koschny, C.M. Soukoulis, E.N. Economou, Phys. Rev. B 75 (2007) 235114.

[20] J.D. Baena, R. Marques, F. Medina, J. Martel, Phys. Rev. B 69 (2004) 014402

[21] D.R. Smith, S. Schultz, P. Markos, C.M. Soukoulis, Phys. Rev. B 65 (2002) 195104.

[22] X. Chen, T.M. Grzegorczyk, B.I. Wu, J. Pacheco, J.A. Kong, Phys. Rev. E. 70 (2004) 016608.

[23] T. Koschny, M. Kafesaki, E.N. Economou, C.M. Soukoulis, Phys. Rev. Lett. 93 (2004) 107402.

[24] User Manual, Version 5.0, CST GmbH, Darmstadt, Germany, http://www.cst.de, 2005. 